\title{
Evaluation of Pleurotus ostreatus as an Ifter Item on Serum Alanine Transaminase (ALT) and Aspartate Transaminase (AST) during Ramadan Fast of Healthy Female Volunteers of Bangladesh
}

\author{
Md. Bazlul Karim Choudhury ${ }^{1}$, Nirod Chandra Sarker ${ }^{2}$, Bimal Chandra Dey ${ }^{3}$, Mst. Masuda Begum ${ }^{4}$, Md. \\ Shahdat Hossain $^{5}$ and M Shahabuddin Kabir Choudhuri ${ }^{6}$
}

${ }^{1}$ PhD. Student, Jahangirnagar University and OSD, DG Health, Mohakhali, Dhaka-1212, Bangladesh. ${ }^{2}$ National Mushroom Development and Extention Center, Sobhanbag, Savar, Dhaka, Bangladesh. ${ }^{3}$ Department of Agriculture Extension, Khamarbari, Dhaka. ${ }^{4}$ Department of Physiology, Shaheed Suhrwardi Medical College, Dhaka, Bangladesh. ${ }^{5}$ Department of Biochemistry and Molecular Biology, ${ }^{6}$ Department of Pharmacy, Jahangirnagar University, Savar, Dhaka, Bangladesh.

\begin{abstract}
In the month of holy Ramadan, food habit of Bangladeshi Muslims grossly changed. During this period they prefer to take lots of oily fried items which might be the cause of hepatocellular injury. The study was undertaken to find out the effect of oyster mushroom (P. ostreatus) on the serum level of hepatocellular enzymes like Alanine Aminotransferase (ALT) and Aspartate Aminotransferase (AST), which are the two common markers for liver function tests (LFTs). The feeding of 50 grams fresh fried oyster mushroom as ifter item in Ramadan fast significantly reduced the serum levels of AST and ALT in female subjects as compared to those with the control females. Findings of the study suggest that $P$. ostreatus may be hepatoprotective in females and hence the human subjects.
\end{abstract}

Key words: P. ostreatus, Ramadan, Ifter ALT, AST.

\section{Introduction}

Mushrooms are basically fungi used not only for consumption but also have medicinal use. There are over 14,000 types of mushrooms in the world, out of which about 3,000 are edible, about 700 have known medicinal properties and around 1400 have been recognized as poisonous ${ }^{1}$.

Different types of mushrooms provide a wide variety of physiological effect in the body. Pleurotus sajo-caju exhibits hypotensive effects which affect the renin-angiotensin system ${ }^{2}$; Trichloma mongolcium produces vasorelaxation because of its lectin content $^{3}$; Pleurotus ostreatus possesses antitumour activity $^{4}$, hepatoprotective activity ${ }^{5}$, antihypertensive activity ${ }^{6}$ and hypoglycaemic effects in experimentally induced diabetes ${ }^{7}$; Lentinus edodes and Grifola frondosa mushrooms have antihypertensive effects in spontaneously hypertensive rats ${ }^{8}$ and Agaricus bisporous decreases serum LDL-C by increasing LDL-C receptor activity'.

Mushroom of Pleurotus species are very much effective in lowering the plasma and liver lipids ${ }^{10,11}$. Lowering of plasma cholesterol reduces the risk of hypercholesterolemia and also improves liver condition.

ALT is a transaminase enzyme which is found in various body tissues, but is most commonly associated with the liver. AST is an enzyme that is raised in the plasma in acute liver damage. It is also found in red blood cells, cardiac muscle, skeletal muscle, the pancreas, and the kidney. Estimation of ALT and AST is known as liver function tests (LFTs) and is used to monitor damage to the liver parenchymal cells. Elevated level of AST is a sign of serious liver damage ${ }^{12}$. ALT is another enzyme associated with liver parenchymal cells. ALT is a more specific indicator of liver damage than the AST, as the AST may also be elevated in diseases affecting other organs. ALT and AST is commonly measured clinically as a part of diagnostic liver function tests, to determine liver health ${ }^{13}$.

This study was carried out to evaluate the effects of Pleurotus Ostreatus as an Ifter item on serum ALT and AST during Ramadan on healthy female volunteers to assess the liver function. It is observed that during iftar Bangladeshi people commonly takes huge amounts of various foods, among them oily and fried dishes are predominate. In this situation this may 
develop health hazard and especially it may create extra load to the liver. On the other hand due to hormonal difference females are more prone to become obese and hence develops various types of liver problem. Mushroom is one of the top priority foods in different countries. In Bangladesh still it is not established as a food. The aim of the study is to evaluate the effect of mushroom on hepatic markers as AST and ALT on fasting female subjects during Ramadan.

\section{Materials And Methods}

The study was conducted during the period of Ramadan in association with Strengthening Mushroom Development Project, National Mushroom Development and Extension Center (NAMDEC), Sobhanbag, Savar, Dhaka.

Subjects: Total 56 subjects were included in the study. They were divided into two groups. In group-1 (G-1), 29 adult female and in group-2 (G-2) age matched 27 female subjects wanting to be fast in the whole Ramadan were considered.

Selection criteria: The Subjects were clarified about the study and after getting their written consent they were included. The details history was taken from the subjects which included age, sex, occupation, educational status, marital status, family history and drug history. Patients suffering from acute illness and disease factors may rise the ALT or AST level and non fast persons were excluded.

Study process: In the study previously divided two groups were included. G-2 was studied without mushroom supplementation. If any drugs previously getting by the subjects, it was continued. 50 grams of fresh $P$. ostreatus was ensured for each individual of G-I by the responsible workers daily by home visits or from the research center. The mushrooms were collected from NAMDEC. At the beginning of Ramadan, subjects were evaluated for health status. Fasting blood sample was collected for analysis. Just after ending of Ramadan the subjects were evaluated and all the investigation procedures were repeated. Serum creatinine ALT and AST were estimated by semi-auto analyzer (3000 evaluation) using commercially available reagent kit.

Statistical analysis: The recorded characteristics of the subjects were analyzed by standard statistical methods using computer software, SPSS package programme.

\section{Results}

Mean $( \pm$ SE) age (years) of G-1 and G-2 were $39.75 \pm 1.59$ and $41.28 \pm 2.60$ ranging from $25-57$ and 25-65 respectively (Fig. 1). No statistically significant mean difference of age of the two groups was seen $(p>0.05)$. Mean $( \pm \mathrm{SE})$ serum creatinine $(\mathrm{mg} / \mathrm{dl})$ level of G-1 and G-2 were $0.69 \pm 0.09$ and $0.65 \pm 0.02$ respectively (Fig. 2). Here also no statistically significant mean difference of creatinine between the two groups was observed $(\mathrm{p}>0.05)$.

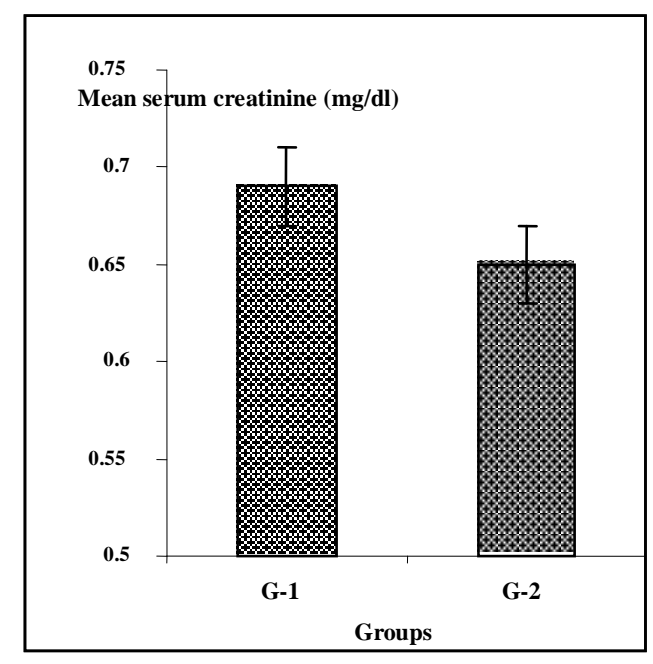

Fig. 1: Mean age of the study groups

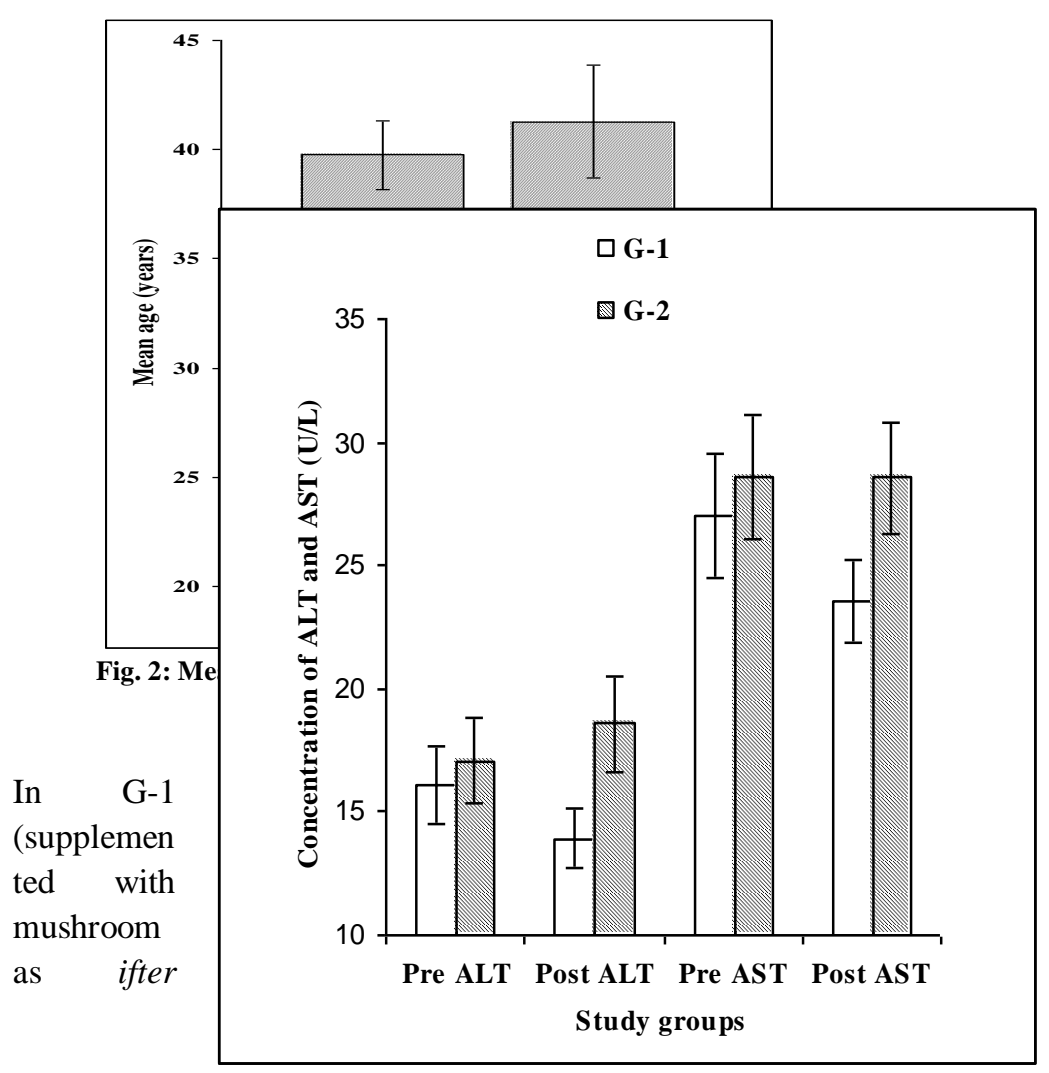


item), the mean $( \pm$ SE) of serum ALT (U/L) before and after Ramadan were $16.10 \pm 1.59$ and $13.93 \pm 1.22$ respectively. A statistically significant mean difference of ALT $(p<0.05)$ was observed in pre and post Ramadan state. The mean $( \pm \mathrm{SE})$ serum level of AST of pre and post Ramadan samples were $27.00 \pm 2.50$ and $23.51 \pm 1.67$ respectively. Here also a statistically significant mean difference between the two periods ( $p<0.05$ ) was observed (Table-1). In G-2 (not supplemented mushroom as ifter item), the mean ( $\pm \mathrm{SE})$ serum ALT (U/L) before and after Ramadan was $17.07 \pm 1.72$ and $18.59 \pm 1.96$ respectively. No statistically significant mean difference of ALT ( $p>0.05$ ) was observed before and after Ramadan. The mean $( \pm$ SE) serum level of AST of pre and post Ramadan samples were $28.62 \pm 2.54$ and $28.55 \pm 2.24$, respectively. Here was also no statistically significant mean difference between the two periods $(\mathrm{p}>0.05)$. In this observation it is seen that there is no reduction of both serum ALT and AST levels at post Ramadan state. Rather a small raise of Serum ALT was seen, although it is not statistically significant (Table-1).

\section{Table 1: Evaluation of serum ALT and AST of G-1 and G-2 subjects}

Results show mean \pm SE. Data were analyzed by Pair $t$ test. Means were significantly different at $\mathrm{p}<0.05$ at $95 \%$ confidence limit.

\section{Fig. 3: Comparison of ALT and AST of pre-and post Ramadan states}

The mean serum levels of ALT of G-1 and G-2 before Ramadan were $16.10 \pm 1.59$ and $17.07 \pm 1.72$ respectively. No statistically significant mean difference $(\mathrm{p}>0.05)$ was observed between the two groups of pre Ramadan state. Whereas after Ramadan those levels were $13.93 \pm 1.22$ and $18.59 \pm 1.96$ respectively (Fig. 3). Here a significant mean difference between the two groups was observed $(\mathrm{p}<0.05)$. Simultaneously, the serum levels of AST of G-1 and G-2 before Ramadan were $27.00 \pm 2.50$ and $28.62 \pm 2.54$ respectively. No statistically significant mean difference ( $\mathrm{p}>$ 0.05 ) between the two groups was observed. But after Ramadan those levels were $23.51 \pm 1.67$ and $28.55 \pm 2.24$. Here a statistically non significant remarkable mean difference $(p=0.07)$ was observed in case of AST levels of G-1 and G-2 (Fig. 3) after Ramadan. These observations indicate that in normal condition both ALT and AST levels in G -1 and G -2 persist in same plan which are significantly reduced by mushroom containing ifter after Ramadan period.

\section{Discussion}

Considering the findings of the study it was observed that there was no significant variation regarding age of the two groups. Serum creatinine level of subjects was estimated to exclude the renal alignment and there was also no significant difference of the study groups. It was observed that supplementation of a considerable amount (50 grams per day) of fried P. ostreatus regularly as ifter item in Ramadan significantly reduced serum ALT and AST in comparison to non mushroom supplemented control subjects. In a study ${ }^{14}$ it was suggested that $5 \% P$. ostreatus supplementation provides health benefits, by acting on the atherogenic lipid profile in the hypercholesterolaemic condition. It is now established that excess lipid accumulation in the liver causes fatty change and ultimately responsible for hepatocellular injury.

The Oyster mushroom contains statins such as lovastatin which works to reduce cholesterol ${ }^{15}$. It had been reported that

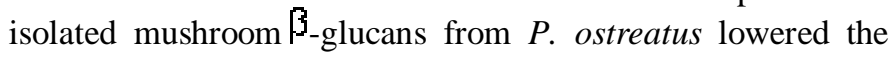
serum cholesterol concentration ${ }^{16}$. It increases the levels of reduced glutathione in the liver and stimulates the activities of catalase and glutathione peroxidase in the liver ${ }^{11}$. In another

\begin{tabular}{ccccc}
\hline \multirow{2}{*}{$\begin{array}{c}\text { Study } \\
\text { groups }\end{array}$} & $\begin{array}{c}\text { Name of the } \\
\text { parameters }\end{array}$ & \multicolumn{2}{c}{ Serum levels (U/L) } & P \\
\cline { 3 - 4 } & & $\begin{array}{c}\text { Pre Ramadan } \\
(\text { Mean } \pm \text { SE) }\end{array}$ & $\begin{array}{c}\text { Post Ramadan } \\
\text { (Mean } \pm \text { SE) }\end{array}$ & \\
\hline G-1 & ALT & $16.10 \pm 1.59$ & $13.93 \pm 1.22$ & $<0.05$ \\
& AST & $27.00 \pm 2.50$ & $23.51 \pm 1.67$ & $<0.05$ \\
G-2 & ALT & $17.07 \pm 1.72$ & $18.59 \pm 1.96$ & $>0.05$ \\
& AST & $28.62 \pm 2.54$ & $28.55 \pm 2.24$ & $>0.05$ \\
& & &
\end{tabular}

study $^{5}$ it was observed that one month supplementation of 50 grams of fried P. ostreatus as ifter item significantly reduced plasma ALT and AST of male subjects at Ramadan.

In another study ${ }^{14}$ observed that administration of the extract of $P$. ostreatus reduces significantly the plasma level of AST, ALT and Alkaline phosphatase (ALP) and increases significantly the hepatic concentration of antioxidant enzymes reduced glutathione (GSH), catalase (CAT), superoxide dismutase (SOD) and glutathione peroxidase (Gpx) on Carbon tetra chloride induced liver damage in male Wister rats. $P$. ostreatus feeding may involve the suppression of endogenous cholesterol biosynthesis by inhibiting the activity of HMGCoA reductase. Mushroom feeding also significantly decreased hepatic cholesterol suggesting the clearance of this sterol component from liver tissue. This may relate to the mushroom-induced enhanced activity of $7 \alpha$-hydroxylase a key enzyme of cholesterol catabolism and bile acid secretion and the subsequent increased excretion of cholesterol through the 
faeces ${ }^{17}$. This study is consistent with ${ }^{15,16}$ which gives the guidelines of hepatoprotective effects of oyster mushroom.

In a literature it was explained that, the mechanism of oyster mushroom's hypocholesterolemic effects lies with its ability to reduce cholesterol absorption and increase plasma cholesterol removal by reducing the production and secretion of very low density lipoproteins (VLDL). Rats were fed with a semisynthetic diet with $0.3 \%$ of cholesterol. $5 \%$ of powdered oyster mushroom was added to the diet for 8 weeks. It was observed that after 8 weeks, the level of serum cholesterol dropped significantly by $36 \%$; and the accumulation of cholesterol and triglycerides in the liver fell by $51 \%$ and $32 \%$, respectively. The researchers explained it as the decreased levels of cholesterol in the blood. Parallel reductions of cholesterol also occurred in VLDL by $53 \%$ and in LDL-C by $47 \%{ }^{17}$.

\section{Conclusion}

Regular consumption of mushroom as an ifter item has a significant role to improve liver function in the fasting female. Although mushrooms are increasingly being recognized as important food products for their significant role in human health, nutrition and disease, their consumption in Bangladesh is extremely limited. One reason probably being that the health benefits derived from various edible mushrooms are largely unknown and lack of mass media focusing to create awareness of the beneficial effects of mushroom which may much more contribute to maintain an optimum health.

\section{References}

1. Annonymousa.http://lifestyle.iloveindia.com/lounge/ benefits of- mushrooms-1337.html.

2. Chang R. Functional properties of edible mushroom. Nutr. Rev. 1996; 54 (Suppl.): S91-93.

3. Wang HX, Ooi VE, Ng TB, Chiu KW, Cang ST. Hypotensive and vasorelaxing activities of a lectin from the edible mushroom Tricholoma mongolicum. Pharmacol. Toxicol. 1996; 79: 318323.

4. Yoshioka Y, Tabeta R, Saito H, Uehara N, Fukuoka F. Antitumor polysaccharides from P. ostreatus (Fr.) Quel. Isolation and structure of a beta-gulcan. Carbohydrate Res. 1985; 140: 93-100.

5. Choudhury MBK, Mowsumi FR, Mujib TB, Sarker NC, Choudhuri MSK, Shahdat M. Effect of Oyster Mushroom (Pleurotus ostreatus) on Hepatocellular Markers Alanin Aminotransferase and Aspartate Aminotransferase of Adult
Human During Ramadan. Bangladesh J. Mushroom. 2009; 3(2): 7-11.

6. Choudhury BK, Amin SMR, Sarkar NC, Khan AS, Mahjabin T, Begum R, Akhtaruzzaman M, Rahman MS. Impact of Oyster Mushroom (Pleurotus Ostreatus) Intake on Hypertension and Blood sugar Status of Common People of Bangladesh. Bangladesh J Med Biochem. 2008; 1(1): 14-17.

7. Chorvathova V, Bobek P, Ginter E, Klvanova J. Effect of the oyster fungus on glycaemia and cholesterolaemia in rats with insulindependent diabetes. Physiol. Res. 1993; 42: 175-179.

8. Kabir Y, Yamaguchi M, Kimura S. Effect of Shiitake (Lentinus edodes) and Maitake (Grifola frondosa) mushrooms on blood pressure and plasma lipids of spontaneously hypertensive rats. $J$. Nutr. Sci. Vitaminol. 1987; 33: 341-346.

9. Fukushima M, Nakano Y, Morii Y, Ohashi T, Fujiwara Y, Sonoyama K. Hepatic LDL receptor mRNA in rats is increased by dietary mushroom (Agraicus bisporous) fiber and sugar beet fiber. J. Nutr. 2000; 130: 2151-2156.

10. Pathak VN, Yadav N, Gaur M. Mushroom Production and Processing Technology. Agrobios (India), Chopasani Road, Jhodhpur, 342002. 1998; p. 179.

11. Opletal L, Jahodár L, Chobot V, Zdansky P, Lukes J, Bratova M, Solichova D, Blunden G, Dacke CG, Patel AV. Evidence for the anti- hyperlipidaemic activity of the edible fungus Pleurotus ostreatus. Br. J. Biomed. Sci. 1997; 54(4): 240-243.

12. Jayakumar T, Ramesh E, Geraldine P. Antioxidant activity of the oyster mushroom, Pleurotus ostreatus, on $\mathrm{CCl}_{4}$-induced liver injury in rats. Food Chem Toxicol. 2006; 44(12):19891996.

13. Alam N, Hossain MS, Khair A, Amin SMR, Khan A. Comparative effects of mushrooms on plasma lipid profile of hypercholesterolaemic rats. Bangladesh J. Mushroom. 2007; 1(1): $15-22$.

14. Annonymous. Alanine transaminase. Internet: en.wikipedia.org/wiki/A lanine_transaminase. 2010a.

15. Annonymous. Aspartate transaminase Internet: en.wikipedia.org/wiki/Aspartate_transaminase. 2010b.

16. Hossain S, Hashimoto M, Choudhury EK, Alam N, Hussain S, Hasan M, Choudhuri SK, Mahmud I. Dietary mushroom (Pleurotus ostreatus) ameliorates atherogenic lipid in hypercholesterolaemic rats. Clin. Exptl. Pharmacol. Physiol. 2003; 30: 470-475.

17. Gunde-Cimerman N, Cimerman A. Pleurotus fruiting bodies contain the inhibitor of 3-hydroxy-3-methylglutaryl-coenzyme A reductase-lovastatin. Exp. Mycol. 1995; 19(1): 1-6. 